\title{
Regulation of apoptosis by a Caenorhabditis elegans BNIP3 homolog
}

\author{
Motoaki Yasuda, Cleta D'Sa-Eipper, Xiu-lan Gong and G Chinnadurai* \\ Institute for Molecular Virology, St Louis University Medical Center, 3681 Park Avenue, St Louis, Missouri 63110, USA
}

\begin{abstract}
We have identified a $C$. elegans protein, ceBNIP3, homologous to the human BCL-2/EIB-19K interacting BCL-2 family pro-apoptotic protein BNIP3. In transiently transfected mammalian cells, ceBNIP3 complexes with CED-9, the worm homolog of BCL-2. CeBNIP3 also efficiently heterodimerizes with the cell death protease proCED-3 by direct binding via the prodomain. Transfection of ceBNIP3 and CED-3 results in enhanced proteolytic processing of the CED-3 zymogen and in cooperative induction of apoptosis. Coexpression of CED-9 suppresses the cooperative cell death induced by ceBNIP3 and CED-3. In cells coexpressing CED-9, ceBNIP3 and CED-3, all three proteins exist as a ternary complex suggesting that CED-9 may suppress cooperative apoptosis induced by CED-3 and ceBNIP3 by simultaneous complex formation with CED-3 and ceBNIP3. Our results suggest that ceBNIP3 may be a novel component of the $C$. elegans apoptosis paradigm and may initiate apoptosis by recruiting CED-3 to mitochondria and other cytoplasmic membranes.
\end{abstract}

Keywords: apoptosis; ceBNIP3; mitochondria; CED-9; CED-3

\section{Introduction}

Genetic studies with the nematode Caenorhabditis elegans, have provided a molecular frame work for the current understanding of the mechanisms underlying apoptosis. Three nematode genes play critical roles in initiation and execution of apoptosis. The Ced9 gene protects cells from cell death while the Ced-4 and the $C e d-3$ genes are required for the execution of cell death (reviewed by Hengartner, 1997). The CED-9 protein is a structural and functional homolog of the mammalian anti-apoptosis protein BCL-2 (Hengartner and Horvitz, 1994). CED-3 is a cysteine death protease (Yuan et al., 1993) and is related to mammalian caspases. Recent biochemical studies have revealed that CED-4 physically interacts with the proCED-3 zymogen and facilitates proteolytic processing of proCED-3 into a functional protease (Chinnaiyan et al., 1997a,b; Seshagiri and Miller, 1997; Wu et al., 1997). CED-9 appears to suppress cell death by simultaneous interaction with CED-4 and proCED-3, thereby preventing the proteolytic processing of CED-3 (Chinnaiyan et al., 1997a).

The mechanism by which the mammalian BCL-2 family proteins suppress apoptosis is not fully resolved. Recent studies have identified a cytoplasmic adapter

*Correspondence: G Chinnadurai

Received 28 July 1998; revised 22 September 1998; accepted 6 October 1998 protein Apaf-1, a human homolog of CED-4 (Zou et al., 1997). Like CED-4, Apaf-1 also associates with a mammalian procaspase, caspase-9 (Zou et al., 1997). In a fashion analogous to CED-9, a mammalian BCL-2 family protein, BCL- $\mathrm{X}_{\mathrm{L}}$ has also been shown to complex with Apaf-1 (Pan et al., 1998; Hu et al., 1998). These results suggest that at least one of the mechanisms by which the mammalian BCL-2 family proteins suppress apoptosis may resemble that of CED-9. The mammalian anti-apoptosis proteins also complex with a number of other proteins (reviewed by Reed, 1997a). Several of these proteins are BCL-2 family pro-apoptotic proteins that share one or more conserved domains with other BCL-2 family members. The pro-apoptotic activity of these proteins appears to be primarily dictated by a single (BH3) conserved domain (Boyd et al., 1995; Chittenden et al., 1995). Human BNIP3 (previously Nip3) was discovered as a BCL-2/E1B-19K binding protein and complexes with several viral and cellular BCL-2 family anti-apoptosis proteins (Boyd et al., 1994; Theodorakis et al., 1996). HuBNIP3 also appears to be a BCL-2 family member since it contains a conserved $\mathrm{BH} 3$ domain and a characteristic C-terminal transmembrane domain (Yasuda et al., 1998) and it is predominantly localized in mitochondria (Boyd et al., 1994; Yasuda et al., 1998; Chen et al., 1997). While most BH3-containing BCL-2 family pro-apoptotic proteins induce rapid cell death, BNIP3 induces a delayed cell death in transfected cells (Yasuda et al., 1998; Chen et al., 1997). Deletion of the BH3 domain of BNIP3 only partially relieves its pro-apoptotic activity (Yasuda et al., 1998), raising the possibility that BNIP3 may promote cell death by certain $\mathrm{BH} 3$-independent mechanisms as well. During databank searches, we discovered that a putative $C$. elegans protein coded an open reading frame ceC14F5.1 (referred to hereafter as ceBNIP3), identified by the $C$. elegans sequencing program, that codes for a protein significantly homologous to human BNIP3. Since the C. elegans apoptosis machinery is simple, we decided to investigate the role of ceBNIP3 in apoptosis in concert with other C. elegans apoptosis regulating proteins. In this communication, we report that ceBNIP3 promotes apoptosis by recruiting and activating the CED-3 protease in a manner similar to CED-4.

\section{Results}

\section{Identification of ceBNIP3}

Data bank searches using BLASTP algorithm (Altschul et al., 1990) revealed that an open reading frame, ceC14F5.1 (referred herafter as ceBNIP3) identified by the $C$. elegans sequencing program codes for a protein significantly homologous to the human BNIP3 (Figure 1a). The homology between these two proteins includes 
a highly homologous 19-aa region (aa 122-140 of ceBNIP3) and the C-terminal trans-membrane domain that is characteristic of BCL-2 family proteins. We cloned the ceBNIP3 ORF by PCR from a genomic cosmid, C14F as well as from a cDNA library. Since huBNIP3 interacts with various mammalian cellular and viral anti-apoptotic BCL-2 family proteins (Boyd et al., 1994; Theodorakis et al., 1996), we examined whether ceBNIP3 interacts with CED-9, the worm homolog of BCL-2 (Hengartner and Horvitz, 1994) by coimmunoprecipitation analysis of proteins expressed in mammalian cells (Figure 1b). BSC-40 cells were transiently transfected with CED-9 (Myc-tagged) and HA-ceBNIP3, metabolically labeled with ${ }^{35} \mathrm{~S}$-methionine/cysteine mixture. Immunoprecipitation with either the $\mathrm{HA} \mathrm{mAb}$ or the $\mathrm{Myc} \mathrm{mAb}$ coprecipitated both CED-9 and ceBNIP3. Although ceBNIP3 was generally expressed at lower levels (compared to CED-9), it was found to be quantitatively coprecipitated with CED-9 (compare the last two lanes of Figure 1b).

\section{Induction of apoptosis by ceBNIP3}

We have previously observed that huBNIP3 induced a delayed cell death activity in transfected mammalian cells while other pro-apoptotic proteins such as BIK induced rapid cell death (Yasuda et al., 1998). We then determined if ceBNIP3 also induces apoptosis in

a
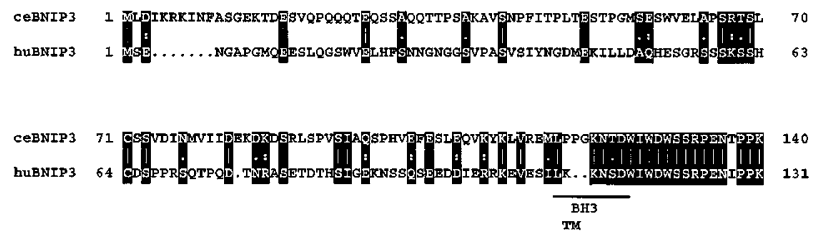

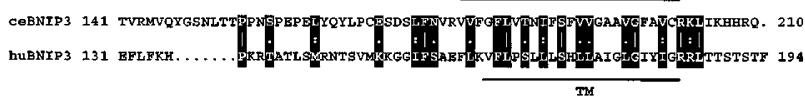

b

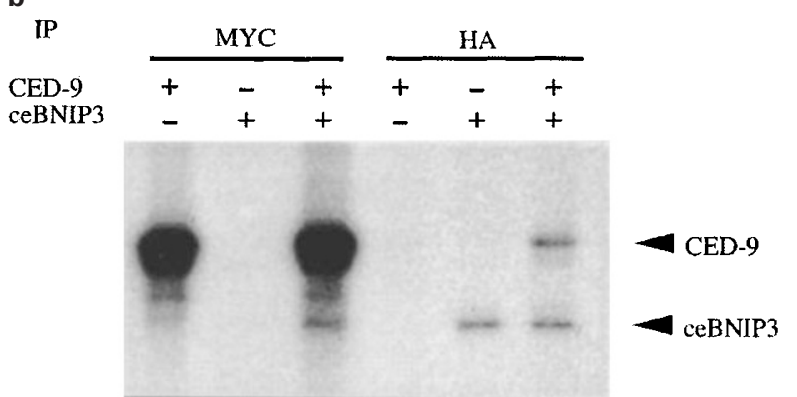

Figure 1 (a) Homology between huBNIP3 and ceBNIP3. The amino acid sequences of huBNIP3 (GenBank accession \#U15174) and ceBNIP3 (GenBank accession \#U29082) were aligned using the GAP program (University Wisconsin GCG package). CeBNIP3 shares $22.2 \%$ identity and $41.8 \%$ similarity with huBNIP3. Identical amino acids are indicated by vertical lines, related amino acids are indicated by two dots and distantly related amino acids are indicated by single dots (within the shaded area). The C-terminal trans-membrane domains of huBNIP3 and ceBNIP3 (indicated as TM) and the BH3 domain of huBNIP3 (Boyd et al., 1994) are underlined. (b) Interaction between ceBNIP3 and CED-9. BSC40 cells were transfected with plasmids expressing HA-ceBNIP3 (HA-tagged) or CED-9 (Myctagged) separately or together. ${ }^{35} \mathrm{~S}$-labeled proteins were immunoprecipitated with the $\mathrm{HA} \mathrm{mAb}$ or the $\mathrm{Myc} \mathrm{mAb}$ and analysed by SDS-PAGE followed by autoradiography transfected mammalian cells. Human 293 cells were transiently transfected with ceBNIP3 or huBNIP3 and apoptosis was quantitated at various times after transfection. In cells transfected with ceBNIP3 apoptosis was detectable at $12-48 \mathrm{~h}$ after transfection (Figure 2). The levels of apoptosis observed in cells transfected either with ceBNIP3 or huBNIP3 at $48 \mathrm{~h}$ after transfection were significantly higher than those observed at earlier times. These results suggest that like huBNIP3, ceBNIP3 also induces delayed cell death albeit at lower levels than huBNIP3.

\section{Interaction with CED-3}

We reasoned that the delayed cell death activity of ceBNIP3 may be due to its recruitment of endogenous death effectors such as caspases. Further, we also observed a modest linear homology between ceBNIP3 and the prodomain of CED-3, the executioner of worm apoptosis (by the SIM alignment program; (Huang and Miller (1991); not shown). We, therefore, examined if ceBNIP3 can interact with proCED-3. BSC-40 cells were transfected with HA-ceBNIP3 and CED-3 wt (Flag) or CED-3G360S (Flag) (a catalytically inactive mutant; Xue et al., 1996) and the metabolically labeled proteins were immunoprecipitated with the Flag $\mathrm{mAb}$ or the HA $\mathrm{mAb}$. CeBNIP3 was efficiently coprecipitated with CED$3(w t)$ or CED-3G360S mutant when immunoprecipitated either with the HA or the Flag mAb (Figure 3a). To determine if the interaction between CED-3 and ceBNIP3 is direct, we examined the binding of in vitro synthesized ( ${ }^{35} \mathrm{~S}$-labeled) CED-3 mutant (G360S) protein with a bacterially expressed and purified GST fusion protein containing ceBNIP3 that lacks the C-terminal trans-membrane domain (GST-ceBNIP3 $\Delta$ TM). CED-3 (G360S) significantly bound to GST-ceBNIP3 $\Delta$ TM compared to GST control (Figure $3 \mathrm{~b}$ ). We also carried out a similar in vitro binding study to determine the interaction between the CED-3 prodomain and ceB-

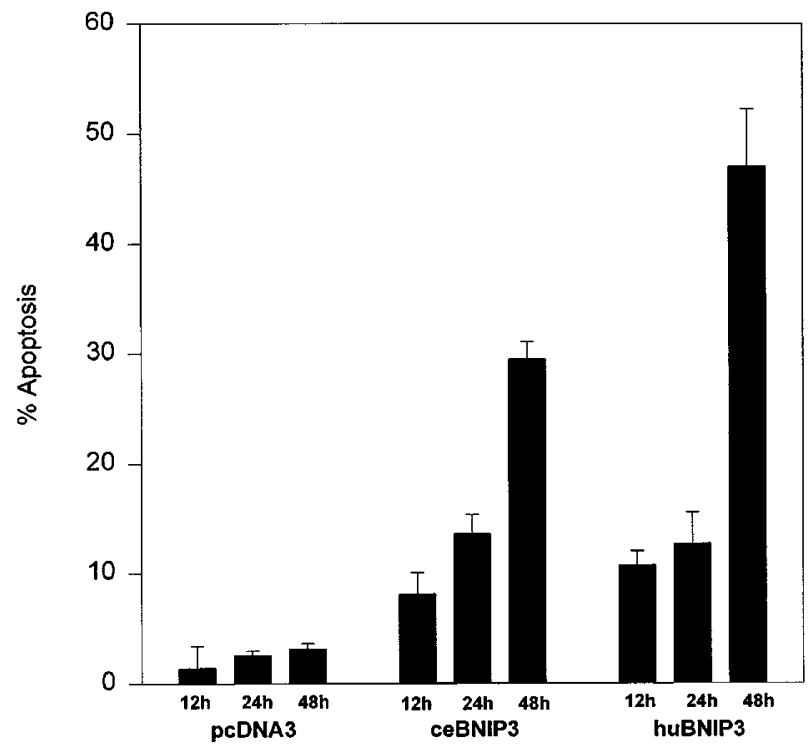

Figure 2 Induction of apoptosis by ceBNIP3 and huBNIP3. Two hundred and ninety-three cells were transfected with plasmids expressing either ceBNIP3 or huBNIP3 along with a reporter plasmid and at various times after transfection percentage of apoptosis was determined as described under Materials and methods 


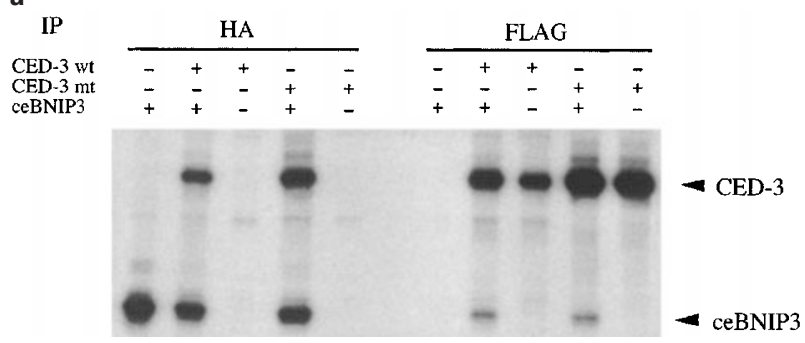

\begin{tabular}{|c|c|c|c|c|c|}
\hline GST-ceBNIP3 $\Delta$ TM & - & - & - & - & + \\
\hline GST & - & - & + & + & - \\
\hline${ }^{35}[\mathrm{~S}] \mathrm{CED}-3 \mathrm{D} 220$ & - & + & - & + & - \\
\hline${ }^{35}[$ S]CED-3 & + & - & + & - & + \\
\hline
\end{tabular}

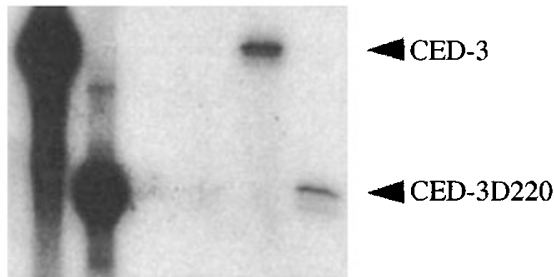

Figure 3 Interaction of ceBNIP3 with CED-3. (a) Coimmunoprecipitation of ceBNIP3 and CED-3. BSC40 cells were transfected with CED-3 (wt or mutant G360S) (Flag) and HAceBNIP3. ${ }^{35}$ S-labeled proteins were immunoprecipitated either with the HA or Flag mAb and analysed by SDS-PAGE and autoradiography. (b) In vitro interaction between ceBNIP3 and CED-3. ${ }^{35}$ S-labeled full-length CED-3 (mutant G360S) or the prodomain (N-terminal 220 aa, prepared from mutant CED3D220) were incubated with the control GST protein or the GSTceBNIP3 $\triangle T$ TM fusion protein and bound proteins were analysed by SDS-PAGE and autoradiography

NIP3. ${ }^{35}$ S-labeled CED-3 prodomain (aa 1-220, prepared from mutant CED-3D220; Wu et al. (1997)) significantly bound to ceBNIP3 $\triangle \mathrm{TM}$ and not the GST control (Figure 3b). Similar results were also obtained by coimmunoprecipitation analysis of mammalian cells transfected with ceBNIP3 and a mutant CED-3 expressing the $\mathrm{N}$-terminal 220 residues (not shown). These results suggest that ceBNIP3 binds directly with CED-3 via the prodomain. We have not investigated if CED-3 has other potential binding sites for ceBNIP3.

\section{Cooperative apoptosis and activation of CED-3}

We then examined if interaction of ceBNIP3 with CED-3 results in any cooperative apoptosis in mammalian cells (Figure 4a). In 293 cells transfected with CED-3 wt, about $13-15 \%$ of the transfected cells underwent apoptosis in a $12 \mathrm{~h}$ period. A similar level of apoptosis was also observed in cells transfected with ceBNIP3. However, cotransfection of both CED-3 wt and ceBNIP3 resulted in a significant increase in apoptosis. In contrast, cotransfection of CED3 mt (catalytically inactive) and ceBNIP3 resulted in lower level of apoptosis compared to ceBNIP3 alone, possibly due to a dominant negative effect of the mutant. The level of cooperative apoptosis induced by ceBNIP3 and CED-3 was comparable to the level of cooperative apoptosis induced by cotransfection of CED-3 and CED-4. We also determined the effect of CED-9 on the cooperative apoptosis induced by

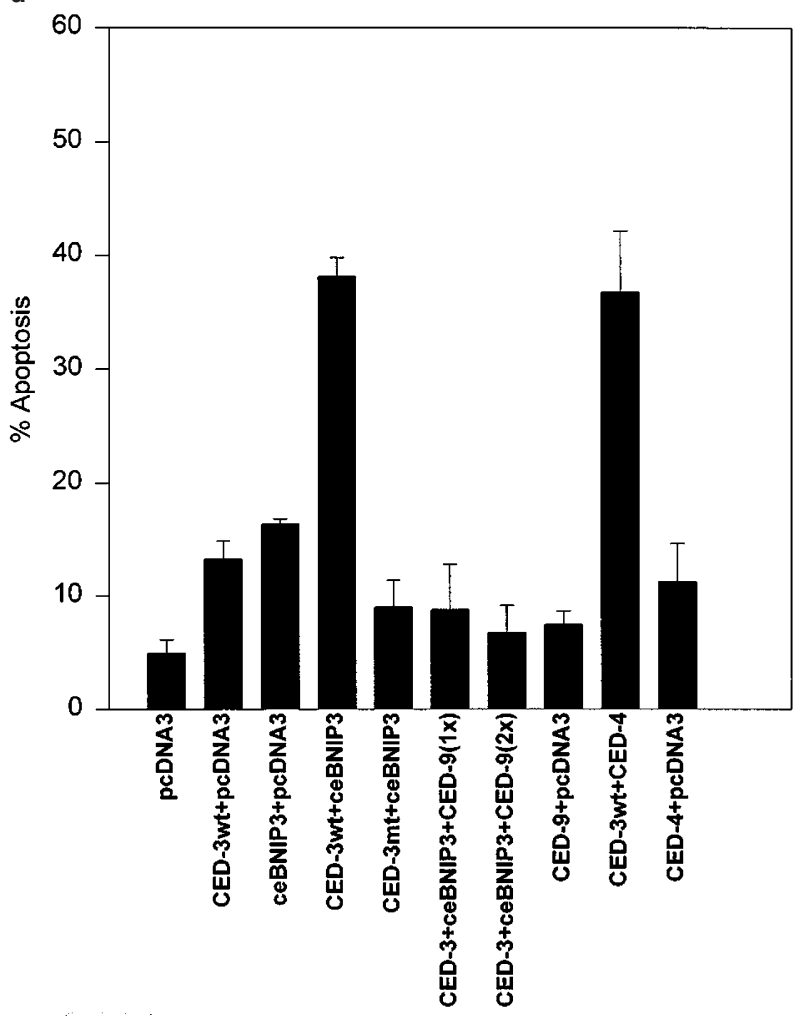

b

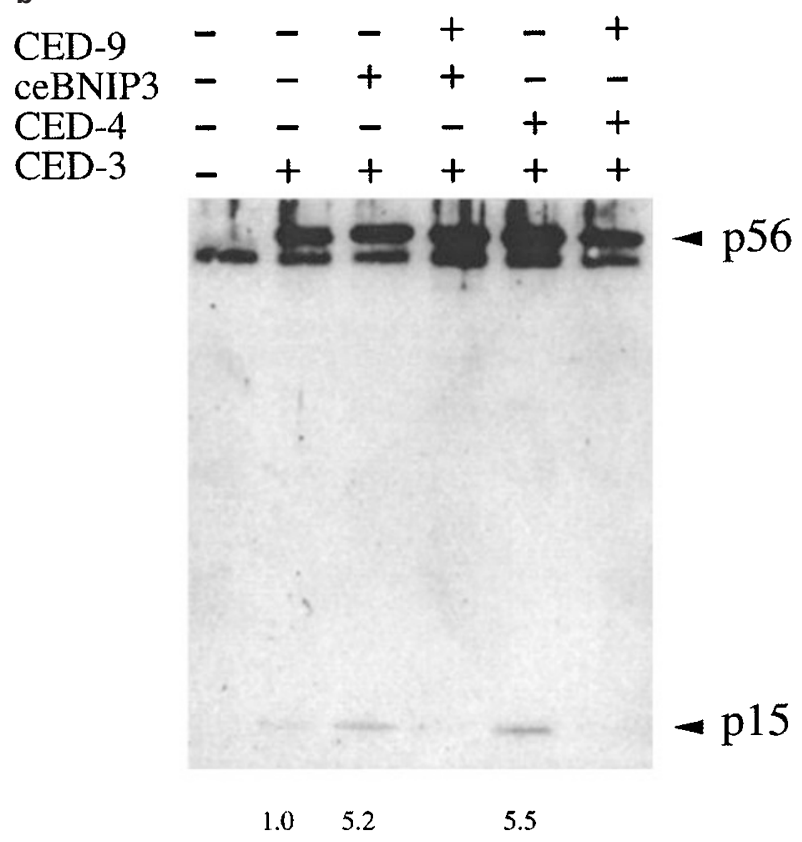

Figure 4 Cooperative apoptosis induced by CED-3 and ceBNIP3. (a) Transient apoptosis assay. Two hundred and ninety-three cells were transfected with plasmids expressing the indicated proteins along with a reporter plasmid and $12 \mathrm{~h}$ after transfection percentage of apoptosis was determined. (b) Effect of ceBNIP3 on processing of CED-3. Two hundred and ninety-three cells were transfected with CED-3 $w t(5 \mu \mathrm{g})$ and ceBNIP3 $(5 \mu \mathrm{g})$ or CED-4 $(5 \mu \mathrm{g})$ or empty vector in the absence or presence of CED-9 $(5 \mu \mathrm{g})$. Twelve hours after transfection, cells were lysed in RIPA buffer, immunoprecipitated with a polyclonal Flag Ab (Santa Cruz Biotechnology) and resolved by SDS-PAGE. The protein blots were probed with a Flag mAb (Kodak). The relative levels of the C-terminal Flag-tagged $15 \mathrm{kDa}$ fragment (p15) of the processed CED-3 relative to total CED-3 were quantitated by densitometry and the values shown at the bottom of the figure. Comparable results were obtained in three independent experiments with s.d. \pm 0.9 for ceBNIP3 and \pm 0.6 for CED- 4 
ceBNIP3 and CED-3. Two different concentrations of CED-9 were cotransfected with ceBNIP3 and CED-3. At both DNA concentrations, CED-9 significantly inhibited apoptosis (Figure 4a). Recently, it has been shown that association between CED-3 and CED-4 enhances the apoptotic activity of CED-3 by facilitating proteolytic processing of proCED-3 (Chinnaiyan et al., 1997a,b; Seshagiri and Miller, 1997; Wu et al., 1997). We, therefore, examined if interaction of ceBNIP3 with CED-3 also enhances the processing of the CED-3 zymogen. Two hundred and ninety-three human cells were transfected with CED-3 (wt) (Flag) alone or along with ceBNIP3. The cell extracts were analysed for the presence of a diagnostic C-terminal p15 (Flag-tagged) fragment generated by proteolytic processing of proCED-3 (Figure 4b). In cells expressing both ceBNIP3 and CED-3 there was significant increase in the level of p15 compared to cells expressing only CED-3. The increase in processing of CED-3 was more or less similar to the increase in processing observed in cells transfected with CED-3 and CED-4. The processing of CED-3 facilitated by ceBNIP3 or CED-4 was strongly inhibited by coexpression of CED-9.

\section{CeBNIP3/CED-3/CED-9 ternary complex}

Since ceBNIP3 binds to both CED-9 (Figure 1b) and CED-3 (Figure 3b) individually, we then asked if CED9 inhibits the cooperative apoptosis by simultaneous binding with ceBNIP3 and CED-3. For this purpose, we cotransfected CED-3G360S (Flag) and HA-ceBNIP3 along with or without CED-9 (Myc). In these studies, we used the mutant CED-3G360S (catalytically inactive) to avoid cell death by CED-3 protease. The cells were metabolically labeled with ${ }^{35} \mathrm{~S}$-methionine/ cysteine mix and the labeled proteins were analysed by immunoprecipitation with the $\mathrm{HA} \mathrm{mAb}$, followed by autoradiography (Figure 5, upper panel) or by Western blot analysis of the immunoprecipitates (Figure 5, lower panels). These results suggest that both CED-9 and CED-3 bind with ceBNIP3 simultaneously, possibly at different sites forming a ternary complex.

\section{Discussion}

We have demonstrated that ceBNIP3 induces apoptosis by recruiting and activating CED-3. Similarly, we have also observed that huBNIP3 associates with certain initiator caspases and induces cooperative apoptosis (unpublished observation). Our results suggest that ceBNIP3 may activate apoptosis by specific recruitment of proCED-3 and enhancing processing of the CED-3 zymogen. This activity of ceBNIP3 is similar to the recently identified activity of CED-4 (Chinnaiyan et al., 1997a; Seshagiri and Miller, 1997; Wu et al., 1997). It remains to be determined if ceBNIP3 initiates an apoptosis paradigm that is independent of CED-4 in $C$. elegans. Ectopic expression of CED-3, in the absence of CED-4, is sufficient to induce apoptosis in C. elegans (Shaham and Horvitz, 1996b). It is possible that ceBNIP3 may play a role in certain auxiliary CED-4independent apoptosis paradigms. As CED-4 is absolutely essential for apoptosis during the worm development (reviewed by Hengartner, 1997), it is also
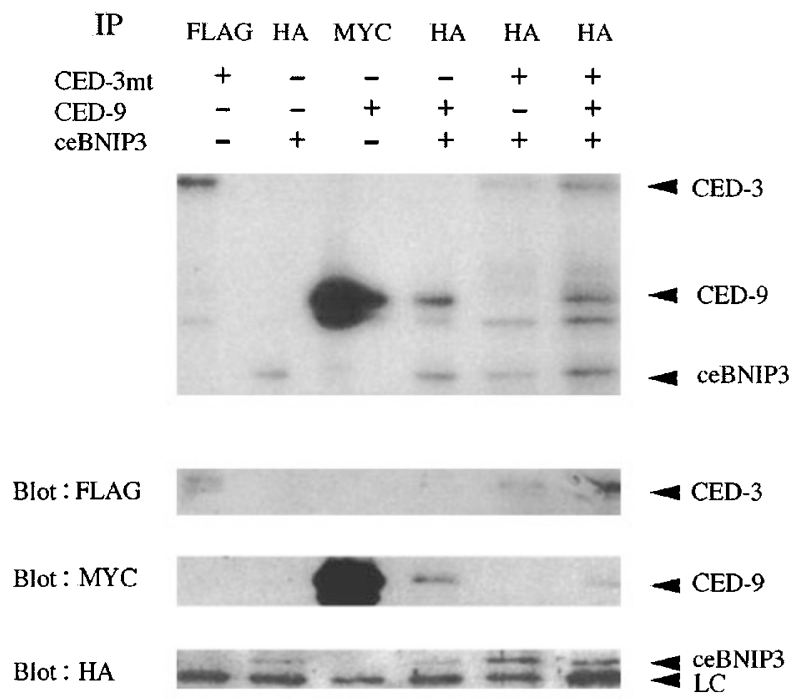

Figure 5 Ternary complex formation by ceBNIP3, CED-3 (G360S) and CED-9. BSC40 cells were transfected with various protein expression plasmids and were metabolically labeled with ${ }^{35} \mathrm{~S}$-methionine/cysteine mix. Proteins were immunoprecipitated with the HA mAb and analysed by SDS-PAGE and autoradiography (upper panel) or the immunoprecipitates were resolved by SDS-PAGE and analysed in a Western blot analysis, probing with the indicated mAb (bottom panels). LC, indicates the background light chain band

possible that the step involving ceBNIP3 may converge into the CED-4 pathway. Since both CED-4 and ceBNIP3 appear to be functionally similar, a model in which apoptosis is initiated by recruitment of CED-3 by ceBNIP3 (specifically to mitochondria and cytoplasmic membrane locales) and subsequent amplification of apoptosis by CED-4 can be envisioned.

HuBNIP3 is primarily a mitochondrial protein and its trans-membrane domain alone can efficiently target heterologous proteins to mitochondria (Yasuda et al., 1998). Since the trans-membrane domain of huBNIP3 and ceBNIP3 are highly homologous, it is possible that ceBNIP3 may also be predominantly a mitochondrial protein. Mitochondrial dysfunction appears to be a dominant theme underlying the early events of apoptosis in mammalian cells (reviewed by Reed, 1997b). However, evidence for mitochondrial involvement in C. elegans apoptosis has thus far been lacking. In mammalian cells, release of cytochrome c from mitochondria appears to play an important role in the onset of an apoptosis pathway that depends on a CED-4 homolog, Apaf1 (Zou et al., 1997). Although it is not known if cytochromes have any apoptotic role in $C$. elegans, it is interesting to note that the cyt-1 gene and the ced-9 gene are linked in a single operon (Hengartner and Horvitz, 1994) suggesting that the expression of these genes may be regulated in a concerted fashion. This evolutionary conservation raises the possibility that cytochromes may also play an apoptotic role in $C$. elegans. It is possible that ceBNIP3 may play a role in early events of $C$. elegans apoptosis by recruiting CED-3 specifically to mitochondria. This may lead to selective proteolysis of mitochondrial substrates resulting in regulated release of certain apoptosis-inducing factors from mitochondria. The intriguing possibility that huBNIP3 and ceBNIP3 play a role in mitochondrial dysfunction 
remains to be elucidated Generation of defined mutants of $C$. elegans in the ceBNIP3 gene would facilitate unraveling its role during worm development. The possibility that ceBNIP3 may be the product of one of the $C$. elegans cell death genes that have previously been identified through genetic analysis (Horvitz et al., 1994) also remains to be investigated.

\section{Materials and methods}

\section{Plasmids}

To construct pcDNA3-HA-ceBNIP3, the $C$. elegans ORF ceC14F5.1 was amplified by PCR using the cosmid C14F as the template and overlapping primers. The PCR product was cloned in a vector pcDNA3-HA. Plasmids pcDNA3CED3 (wt) (Flag), pcDNA3-CED3(G360S)(Flag) and pcDNA3-CED3D220 (AU1) were gifts from Gabriel Nunez and are described in Wu et al. (1997). PcDNA3CED9 (Myc) was constructed by cloning the CED-9 ORF from the cDNA clone B30 (Yuan et al., 1993) (gift from Michael Hengartner) in pcDNA3.1 (Invitrogen). pcDNA3CED4 (Flag) was constructed by cloning the CED-4 ORF (Shaham and Horvitz, 1996a) (gift from Robert Horvitz) in pcDNA3.1.

\section{Immunoprecipitation and Western blot analyses}

BSC40 cells $\left(2 \times 10^{6}\right.$ cells $)$ were transfected with pcDNA3based protein expression plasmids $(3-5 \mu \mathrm{g})$ using the LipofectAmine reagent (GIBCO/BRL). To enhance the level of protein expression, transfected cells were infected with vaccinia virus vTF7-3 (10 p.f.u./cell) that expresses the T7 RNA polymerase (Ausubel et al., 1992). The cells were pulse-labeled with ${ }^{35} \mathrm{~S}$-methionine-cysteine mix (EX$\mathrm{PRE}^{35} \mathrm{~S}^{35} \mathrm{~S}$, NEN, $\left.150 \mathrm{Ci} / \mathrm{ml}\right)$ between $18-20 \mathrm{~h}$ post infection/transfection. Cells were lysed in TNN $(40 \mathrm{~mm}$ Tris-Cl pH 7.5, $150 \mathrm{mM} \mathrm{NaCl}, 0.1-0.5 \% \mathrm{NP} 40,0.04 \mathrm{mg} /$ $\mathrm{ml}$ aprotonin, $0.2 \mathrm{mg} / \mathrm{ml}$ leupeptin and $200 \mu \mathrm{M}$ PMSF), immunoprecipitated with various monoclonal antibodies and analysed by SDS - PAGE. In addition to BSC40 cells, 293 cells were also used to express the various proteins (without vTF-7 infection). These cells were lysed in RIPA buffer (150 mM NaCl, $50 \mathrm{~mm}$ Tris-Cl (pH 8.0), 1\% NP40, $0.5 \%$ sodium deoxycholate and $0.1 \%$ SDS) containing

\section{References}

Altschul SF, Gish W, Miller W, Myers EW and Lipman DJ. (1990). J. Mol. Biol., 215, 403-410.

Ausubel FM, Brent R, Kingston R, Moore D, Seidman J, Smith J and Struhl K. (1992). Current Protocols in Molecular Biology. John Wiley and Sons: New York.

Boyd JM, Marstrom S, Subramanian T, Venkatesh LK, Schaeper U, Elangoban B, D'Sa-Eipper C and Chinnadurai G. (1994). Cell, 79, 341-351.

Boyd JM, Gallo GJ, Elangoban B, Houghton AB, Malstrom S, Avery BJ, Ebb RG, Subramanian T, Chittenden T, Lutz R and Chinnadurai G. (1995). Oncogene, 11, $1921-1928$.

Chen G, Ray R, Dubik, Shi LF, Cizeau J, Bleackley RC, Saxena S, Gietz RDC and Greenberg AH. (1997). J. Exp. Med., 186, $1975-1983$

Chinnaiyan AM, O'Rourke K, Lane BR and Dixit VM. (1997a). Science, 275, $1122-1126$.

Chinnaiyan AM, Chaudhary D, O'Rourke K, Koonin EV and Dixit VM. (1997b). Nature, 388, $728-729$.

Chittenden T, Flemington C, Houghton AB, Ebb RG, Gallo GJ, Elangovan B, Chinnadurai G and Lutz RJ. (1995). EMBO J., 14, 5589-5596. protease inhibitors. Total cell lysates were clarified and precleared with protein A-Sepharose for $1 \mathrm{~h}$. Clarified lysates or immunoprecipitates were run on SDS-PAGE and transferred to nitrocellulose membranes. The membranes were probed with indicated Abs and detected using chemiluminescence (Amersham LIFESCIENCES) detection system.

\section{In vitro protein binding}

${ }^{35}$ S-labeled full-length CED-3 (G360S) and the prodomain were prepared by in vitro transcription and translation of pcDNA3-CED3(G360S) and pcDNA3-CED-3D220 using a commercially available kit (Promega). The labeled proteins were incubated with GST or GST-ceBNIP3 $\Delta$ TM fusion protein immobilized on glutathione-agarose beads (Sigma) in a buffer containing $50 \mathrm{~mm}$ Tris $(\mathrm{pH} \mathrm{7.4),} 150 \mathrm{~mm} \mathrm{NaCl}$, $1 \mathrm{~mm}$ EDTA, $0.2 \% \mathrm{NP} 40$, washed five times in the same buffer and analysed by SDS-PAGE.

\section{Apoptosis assay}

Two hundred and ninety-three cells $\left(3-4 \times 10^{4} /\right.$ well in 12 well plates) were transfected with $0.5 \mu \mathrm{g}$ of the plasmid expressing the individual pro-apoptosic protein and $0.2 \mu \mathrm{g}$ of pCMV- $\beta$ gal reporter plasmid using the LipofectAmine reagent. Total DNA concentration per well was adjusted to $2.0 \mu \mathrm{g}$ with empty vector. Twelve hours after transfection, cells were fixed, stained with X-gal and microscopically examined. One hundred to 200 blue color cells were microscopically scored as apoptotic (round and fragmenting) or non-apoptotic (flat).

\section{Acknowledgements}

We thank Gabriel Nunez, Robert Horvitz, Michael Hengartner, John Spieth, Alan Coulson and Robert Barstead for the gift of various expression constructs and the DNA used in the construction of other expression vectors. We also thank B Elangovan for his help in sequence alignments. We thank Bob Lutz and Tom Chittenden for comments on the manuscript. This study was supported by research grants CA-33616 and CA-73803 from the National Cancer Institute and VM-174 from the American Cancer Society.

Hengartner MO. (1997). Nature, 388, 714-715.

Hengartner MO and Horvitz HR. (1994). Cell, 76, 665-676.

Horvitz HR, Shaham S and Hengartner MO. (1994). Cold Spring Harb. Symp. Quant. Biol., 59, 377-385.

$\mathrm{Hu} \mathrm{Y}$, Benedict $\mathrm{AM}, \mathrm{Wu} \mathrm{D}$, Inohara $\mathrm{N}$ and Nunetz $\mathrm{G}$ (1998). Proc. Acad. Sci. USA, 95, 4386-4391.

Huang X and Miller W. (1991). Adv. Applied Math., 12, $337-357$

Pan G, O'Rourke K and Dixit VM. (1998). J. Biol. Chem. 273, 5841-5845.

Reed JC. (1997a). Nature, 387, 773-776.

Reed JC. (1997b). Cell, 91, 559-562.

Shaham S and Horvitz HR. (1996a). Cell, 86, 201-208.

Shaham S and Horvitz HR. (1996b). Genes and Dev., 10, $578-591$.

Seshagiri S and Miller LK. (1997). Curr. Biol., 7, 455-460.

Theodorakis P, D'Sa-Eipper C, Subramanian T and Chinnadurai G. (1996). Oncogene, 12, 1707-1713.

Wu DY, Wallen HD, Inohara $\mathrm{N}$ and Nunez G. (1997). $J$. Biol. Chem., 272, $21449-21454$. 
Xue D, Shaham S and Horvitz HR. (1996). Genes and Dev., 10, $1073-1083$.

Yasuda M, Theodorakis P, Subramanian T and Chinnadurai G. (1998). J. Biol. Chem., 273, 12415-12421.
Yuan J, Shaham S, Ledoux S, Ellis HM and Horvitz HR. (1993). Cell, 75, 641-652.

Zou H, Henzel WJ, Liu XS, Lutschg A and Wang XD. (1997). Cell, 90, 405-413. 